\title{
Investigation on the Mechanism of Drag Modification over Triangular Riblets
}

\author{
M. M. Zhang ${ }^{1,2,3,4 \dagger}$ Z. L. Zhang ${ }^{1,2,3,4}$ and M. Zhao ${ }^{5}$ \\ ${ }^{1}$ Institute of Engineering Thermophysics, Chinese Academy of Sciences, Beijing 100190, China \\ ${ }^{2}$ Key Laboratory of Wind Energy Utilization, Chinese Academy of Sciences, Beijing 100190, China \\ ${ }^{3}$ University of Chinese Academy of Sciences, Beijing 100049, China \\ ${ }^{4}$ Dalian National Laboratory For Clean energy, Chinese Academy of Sciences, Dalian 116023, China \\ ${ }^{5}$ Department of Mechanics, School of Mechanical Engineering, Tianjin University, Tianjin 300350, China \\ †Corresponding Author Email: mmzhang@iet.cn
}

(Received June 30, 2019; accepted December 2, 2019)

\begin{abstract}
This paper presents experimental and numerical investigations on the modification of local spanwise skinfriction over triangular riblets under the total drag reduction condition. Specifically, the mean and fluctuating vortical flow fields were measured using 2-components X-wires and computed using LES, respectively. Besides, the relationship between local skin-friction along the riblet spanwise and associated vortex evolution was also built using the vortex dynamic method. Based on these results, it was found that, compared with the smooth case, the impaired and enhanced vortex strength, and resultant viscous diffusion/energy dissipation, determined the reduction and augment of the viscous drag force over the local spanwise riblet groove, i.e., decreasing and increasing cases of local drag, respectively. Furthermore, the mean normal diffusion fluxes of normal and spanwise vorticities contributed more to the viscous drag under these two cases. Correspondingly, the relevant flow physics related to these phenomena was discussed in detail.
\end{abstract}

Keywords: Riblets, Drag reduction; Local skin-friction; Vortex dynamic method.

\section{NOMENCLATURE}

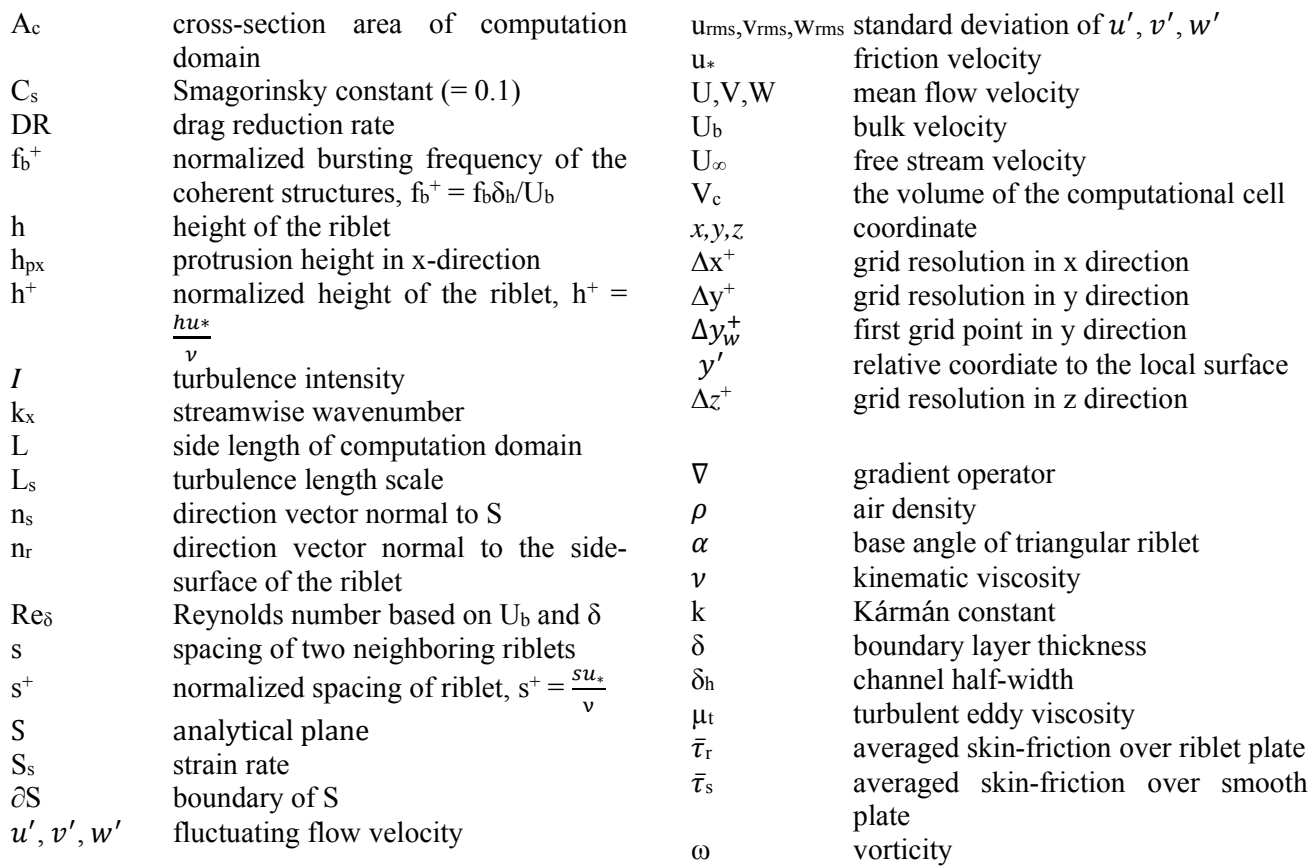


M. M. Zhang et al. / JAFM, Vol. 13, No. 4, pp. 1093-1106, 2020.

$\sigma \quad$ boundary vorticity flux

$\lambda_{x}, \lambda_{y}, \lambda_{z} \quad$ 3D scale size of LES computation

\section{INTRODUCTION}

As we know, the drag reduction on turbulent flow over many military and civilian structures, e.g. airplanes, steamships, automobiles, natural gas/oil pipelines, high-speed railway trains and wind turbine blades, has both academic and engineering significance, and it has been a matter of primary interest for more than half a century. Meanwhile, with the fast development of bionics in recent years, some potential anti-drag solutions based on bionics have been proposed. Among them, streamwise aligned microgrooves or riblets, mimicking shark skin, have been widely acknowledged as a practical choice for reducing skin-friction in the aforementioned structures (Luo et al. (2016)).

A large amount of research work on the viscous drag reduction of riblets has been conducted in the past, and various riblet parameters, i.e., cross-section shape, scale (spacing $\mathrm{s}$ and height $\mathrm{h}$ ) and directivity (yaw angle), and flow pressure gradient (Viswanath (2002), Dean and Bhushan (2010), Yu et al. (2018), Heidarian et al. (2018)), have been examined. The maximum decrease in skin-friction could reach up to around $10 \sim 15 \%$ at an optimum dimensionless rib height $\mathrm{h}^{+}\left(=\mathrm{hu}_{*} / \mathrm{v}, \mathrm{h}\right.$ and $v$ stand for friction velocity and kinematic viscosity, respectively) of the range $8 \sim 18$.

As for the corresponding flow mechanism, some investigations have also been carried out, including $2 \mathrm{D}$ and $3 \mathrm{D}$ cases. In terms of the $2 \mathrm{D}$ case, the riblets were primarily regarded as the typical 2D longitudinal grooved structures. So far, four kinds of explanations about the drag reduction mechanism of riblets have been publically re-ported: (1) spanwise inhibition, i.e., the spanwise movements of streamwise vortices were inhibited by riblets, which impaired the turbulent momentum transport near the wall surface and thereby reduced the skin-friction (Choi (1989), Chu and Karniadakis (1993), Monfared et al. (2019)); (2) existence of protrusion height, i.e., the existence of the distance between virtual origin and riblet tip suppressed the spanwise shift of the viscous sublayer where the riblets were submerged, resulting in more stable low-speed streaks and lower bursting frequencies (Bechert and Bartenwerfer (1989), Luchini et al. (1991), Grü neberger and Hage (2011));(3) creation of a secondary vortex, i.e., a couple of secondary vortices generating near the riblet tip weakened the main streamwise vortical flow in the turbulent boundary layer, which retarded the strong interactions among neighboring streaks and subsequently kept a quasistatic flow in rib valley (Chu and Karniadakis (1993), Bacher and R. Smith (1986), Boomsma and Sotiropoulos (2016)); (4) uplifting of streamwise vortex, i.e., the ribs might uplift the streamwise vortices to decrease the ejection and sweeping events which were related with the high shear stress force in 甲uu energy of streamwise fluctuating
velocitie turbulent flows. Thus, the viscous drag force was reduced (Lee and Lee (2001), Martin and Bhushan (2014), Huang et al. (2016)). As for the 3D case, a real shark skin morphology was modeled and the interpretations on the drag reduction mechanism were more complicated, including the inhibition effect of micro/nano structured morphology on the turbulence, the influence of scale's attack angles, nano-long chains and the boundary layer slipping due to the super-hydrophobicity (Chen et al. (2014), Wen et al. (2014), Boomsma and Sotiropoulos (2016)).

Even so, the physical mechanism of the drag reduction by riblets has still been inconclusive. One main reason is the fact that most previous research only focused on the understanding of the riblet effect on the total viscous drag over the wall surface, while few discussed the effects of the microscopic flow structures around riblets, especially the vortex structures concerned with the evolving flow field, on the local distribution of skin-friction (Bechert et al. (1997), García-Mayoral and Jiménez (2011), Martin and Bhushan (2014), Benschop and Breugem (2017)). Further investigating these ignored points will help to get a better understanding on the drag reduction mechanism of riblets. Therefore, the corresponding research is urgently needed.

In the present paper, for the initial step, the $2 \mathrm{D}$ triangular riblets were chosen to investigate the control of skin-friction within the fully developed turbulent boundary layer via experimental and numerical methods. Since real shark skin is much more complex than the riblets, our future studies will focus on different riblet configurations, such as riblet cross-section shape, orientation etc. First, the detailed spanwise variation of the viscous drag in the riblet grooves was obtained through hot-wire experiment. Meanwhile, the CFD computation was performed and verified to describe the corresponding flow field around the riblets. Based on these results, together with the vortex dynamic method, a quantitative relationship between local wall skinfriction at different spanwise locations and neighboring turbulent flow field was built to elucidate the mechanism behind the drag modification of the riblets from a new perspective.

\section{EXPERIMENTAL SETUP}

All the experimental work was carried out in an open-loop wind tunnel with a test section of $0.5 \mathrm{~m}$ (width) $\times 0.5 \mathrm{~m}$ (height) $\times 4 \mathrm{~m}$ (length) at the Institute of Engineering Thermophysics, Chinese Academy of Sciences. The stable flow speed in the test section was in the range of $1 \sim 40 \mathrm{~m} / \mathrm{s}$, and both the maximum non-uniformity and longitudinal tubulence intensity were under $0.4 \%$.

A smooth support plate $(0.50 \mathrm{~m}$ (width) $\times 2.30 \mathrm{~m}$ 


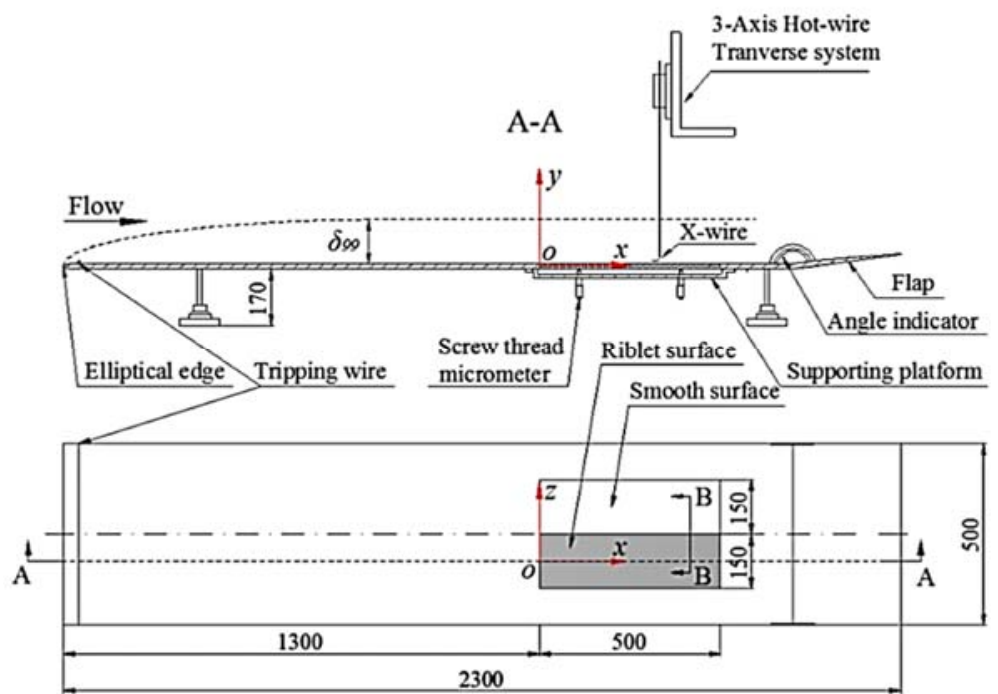

(a)

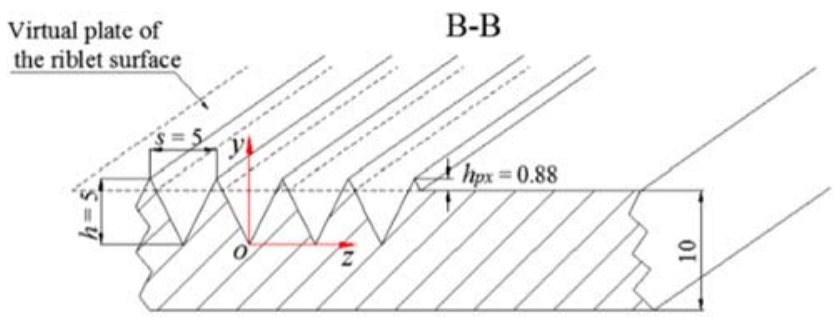

(b)

Fig. 1. Experimental setup: (a) sketch of test system; (b) cross-section of triangular riblet plate. Unit:

mm.

(length) $\times 0.01 \mathrm{~m}$ (thickness)) was horizontally installed $0.5 \mathrm{~m}$ downstream from the test section and $0.17 \mathrm{~m}$ away from the bottom wall of the wind tunnel, as shown in Fig. 1(a). The elliptical shape at the leading edge and the adjustable flap at the trailing edge were used to prevent flow separation from the plate. To stimulate the turbulent boundary layer transition, a trip wire was placed near the leading edge of the plate.

A test plate $(0.30 \mathrm{~m}$ (width) $\times 0.50 \mathrm{~m}$ (length) $\times 0.01$ $\mathrm{m}$ (thickness)) was flush mounted at $1.30 \mathrm{~m}$ from the leading edge of the support plate. Pitot probes were used to measure the wall pressures along five streamwise positions. The corresponding data acquisition frequency was $200 \mathrm{~Hz}$ and the sample length was $10 \mathrm{~s}$. By adjusting the inclination angle of the plate using four high-precision micrometers, the pressures along the streamwise positions kept constant, implying that the turbulent flow was under the zero pressure gradient condition (Marusic et al. (2015), Tang et al. (2016)). In order to keep a constant incoming flow, the test plate was di-vided into two halves [see Fig. 1(a)], i.e., a smooth plate and a riblet plate. The riblet plate was com-posed of grooves with an isosceles-triangle cross-section $(h=$ $s=5 \mathrm{~mm}$ ), indicated in Fig. 1(b). In this study, the streamwise, wall-normal and span-wise coordinates were denoted by $\mathrm{x}, \mathrm{y}$ and $z$, respectively, with the origin locating in the rib valley. The three corresponding-coordinate components of mean and fluctuating velocities were denoted by $U, V, W$ and $u^{\prime}, v^{\prime}, w^{\prime}$, respectively. The leading edge of the riblet plate was sharp. According to our test, this kind of sharp leading edge had little influence on the turbulent flow and the drag reduction by measuring and comparing the profiles of mean and fluctuating flow velocities at the leading edge and several other downstream positions.

The experimental research was conducted under the optimal drag reduction condition, where the influences of the riblets on the boundary layer flow were the most evident. For this purpose, the incoming free stream velocity $\left(U_{\infty}\right)$ was set at 1.02 $\mathrm{m} / \mathrm{s}$. The inflow conditions, specifically, profiles of the mean velocity and the turbulence intensities at the streamwise position of $\mathrm{x}=0 \mathrm{~mm}$ were shown in Fig. 2. It should be noted that $y^{\prime} / \mathrm{h}$ denoted the nondimensional relative height to the smooth surface (as the origin of the experimental coordinate was set at the riblet valley, shown in Fig. 1, we used $y^{\prime}$ represented the relative height to the local surface in this paper). Turbulent intensities $\left(I_{u}, I_{v}, I_{w}\right)$ were defined as the ratio of the standard deviation of the fluctuating velocities, i.e., $u_{r m s}, v_{r m s}, w_{r m s}$, to the free stream velocity $U_{\infty}$. The fully developed turbulent flow was confirmed as the flow behind $\mathrm{x}=0 \mathrm{~mm}$ 
exhibited identical mean velocity and turbulent intensity profiles. Under these setups, the normalized $\mathrm{h}^{+}$and $\mathrm{s}^{+}$were about 15.8, giving rise to a total drag reduction percentage of $6.23 \%$, which was close to the result of Walsh and Lindemann (6.70\%) as $\mathrm{h}^{+}=\mathrm{s}^{+}=15$ (Walsh and Lindemann (1984)). Correspondingly, the Reynolds numbers $(\operatorname{Re} \delta)$ based on the turbulent boundary layer thickness $(\delta)$ and bulk velocity $\left(\mathrm{Ub}_{\mathrm{b}}=1 / \mathrm{A}_{c} \int_{\mathrm{A}_{c}} \mathrm{UdA}_{c}\right)$ over the smooth and riblet plates, i.e., $\operatorname{Re}_{\delta}=\mathrm{U}_{b} \delta / v$, were 6884 and 6835 , respectively. Here, $\delta$ was measured at $\mathrm{x}=350$ $\mathrm{mm}$ (fully developed turbulent region). Unless otherwise stated, the experimental data was all measured at $x=350 \mathrm{~mm}$. In addition, in order to avoid the extra crosswise flow in the test plate, the origin of smooth surface was located at the "virtual origin" of the riblet surface which was the position where the boundary layer 'sees' the riblets as an equivalent smooth wall $\left(\mathrm{h}_{\mathrm{px}}\right.$, as illustrated in Fig. 1(b), was the longitudinal protrusion height between the riblet tip and the virtual origin (Bechert and Bartenwerfer (1989))). This setup was also used in other previous studies to avoid the extra crosswise flow (Lee and Lee (2001), Lee and Choi (2008)).

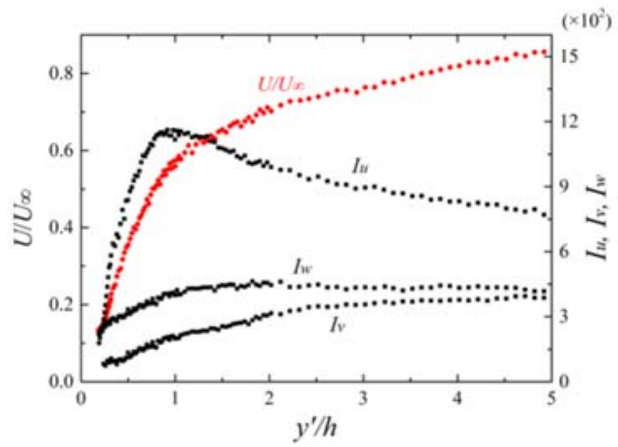

Fig. 2. Inflow conditions: the mean velocity and the turbulence intensities at the streamwise position $x=0 \mathrm{~mm}$.

The mean and fluctuating velocities were measured using the tungsten $\mathrm{X}$-wires with the diameter of 3.8 $\mu \mathrm{m}$ (TSI-1240 for $\mathrm{u}-\mathrm{w}$ measurement) and $25.4 \mu \mathrm{m}$ (TSI-1249A for $\mathrm{u}-\mathrm{v}$ measurement), which were specifically designed for the boundary layer test. The constant-temperature anemometer (TSI IFA- 300CTA) was used and the $\mathrm{X}$-wires were operated at an overheat ratio of 1.4. To accurately adjust the movement of the X-wires within the thin sublayer over the wall surface, a high-precision 3DNEWPORT coordinate transfer system, with the minimum step-sizes in $\mathrm{x}, \mathrm{y}$ and $z$ directions of 1.5 $\mu \mathrm{m}, 10 \mu \mathrm{m}$ and $10 \mu \mathrm{m}$, was utilized. With the assistance of the stereoscopic microscope, the Xwires were precisely positioned on the riblet peak or the smooth plate. This reference position was then utilized to move the probes away from the surface. During our $\mathrm{X}$-wire experiments, both $\tau_{\mathrm{s}}$ and $\tau_{\text {r, }}$, i.e., the local viscous drag force over the smooth and riblet plates, were calculated by linear-fitting the velocity profiles within viscous sublayer. Specifically, about 20 data points in $y^{1+} \leq 6$ were deployed, with the minimum distance of $0.02 \mathrm{~mm}$ from the wall. In these experiments, the measurement uncertainty of the mean and fluctuating velocities by $\mathrm{X}$-wires was estimated to be smaller than $1.0 \%$ according to the Bentley method (Bentley (2004)). Signals from $X$-wires were conditioned and digitized using a 16-bit A/D board at a sampling frequency of $10.0 \mathrm{kHz}$ per channel. The du-ration of each record was about $52.0 \mathrm{~s}$.

\section{NUMERICAL SCHEMES}

\subsection{CFD Schemes}

Besides the experimental study, a numerical investigation was also conducted under the same flow conditions by using the ANSYS FLUNENT 15.0 software. In order to implement the simulations, a cubic computation domain with the side lengths $L_{x}$, $\mathrm{L}_{\mathrm{y}}$ and $\mathrm{L}_{\mathrm{z}}$ of $1000 \mathrm{~mm}, 320 \mathrm{~mm}$ and $320 \mathrm{~mm}$ was built up. This setup corresponded to $\mathrm{x}-$ and $z$ domain size $\left(\lambda^{+}\right.$and $\lambda^{+}$, normalized by $\mathrm{u}^{*}$ and $v$ ), of 3267 and 1045 wall units, satisfying the mini-mum scale requirements (300 and 100 wall units in $\mathrm{x}$ - and $z$-directions) for investigating wall turbulent flows (Jiménez and Moin (1991), Martin and Bhushan (2014)). In addition, a smaller computa-tional domain was used to study the effects of ri-blets in previous studies (Choi et al. (1993), Martin and Bhushan (2014), Ng et al. (2016)). Obviously, it was reasonable that the present domain size was sufficient to properly capture the turbulent flow over smooth and riblet wall surfaces. For the sake of simplification, streamwise riblets with the same cross-section size as their experimental counterparts, were placed on the bottom surface while the upper surface was smooth for comparison, as shown in Fig. 3(a). In our simulation, the top and bottom surfaces were set as no-slip wall boundary conditions, and the left and right surfaces, together with that in the streamwise direction, were set as periodic boundary conditions. The simulation was carried out under a constant mass flow rate $\mathrm{Q}\left(=\mathrm{A}_{c} \int_{\mathrm{A}_{c}} \mathrm{UdA}_{c}\right)$ of 0.0934 $\mathrm{m} 3 / \mathrm{s}$. Correspondingly, the Reynolds numbers $\left(\operatorname{Re}_{\delta}\right)$ based on $\mathrm{U}_{\mathrm{b}}$ and $\delta$ ( $\delta$ was calculated at $\mathrm{x}=350 \mathrm{~mm}$, same as the experiment), were 6887 and 6829 for the smooth and riblet plates, respectively. It should be noted that the asymmetric mean velocity profile and reduced effective channel height would be created by introducing the riblets on one surface. However, by adopting this setup, the same incoming flow conditions as the smooth plate case could be kept for the riblet case. In addition, both $\bar{\tau}_{s}$ and $\bar{\tau}_{r}$ were acquired using the velocity gradients of viscous sublayer, same as the experimental method.

To capture the unsteady flow structures within the turbulent boundary layer, the Smagorinsky-Lilly subgrid-scale turbulence model (Martin and Bhushan (2014)) was adopted in our LES simulation to mimic the behavior of near-wall subgrid stress and turbulent eddy viscosity, expressed as $\mu_{\mathrm{t}}=\rho \mathrm{L}_{\mathrm{s}}{ }^{2}\left|\mathrm{~S}_{\mathrm{s}}\right|$. Here, $\rho, S_{s}$ and $L_{s}$ were the air density, strain rate and turbulence length scale $\left(\mathrm{L}_{\mathrm{s}}=\min \left(\kappa_{\mathrm{y}}, \mathrm{C}_{\mathrm{s}} \mathrm{V}_{\mathrm{c}}{ }^{1 / 3}\right)\right)$, where $\mathrm{K}$ á rmá $\mathrm{n}$ constant was $\kappa=0.41$, Smagorinsky constant was $\mathrm{C}_{\mathrm{s}}=0.1$, and $\mathrm{Vc}$ was the volume of the 
computational cell. Accordingly, the second-order scheme for spatial discretization and bounded central differencing scheme for momentum terms were selected to eliminate numerical errors. In order to decouple static pressure and velocity, the SIMPLEC method was utilized to handle unsteady incompressible Navier-Stokes equations at a time step of $0.001 \mathrm{~s}$ with 30 sub-iteration steps $\left(\Delta \mathrm{t}^{+}=\right.$ $\left.\Delta \mathrm{tU}_{\mathrm{b}} / \delta_{\mathrm{h}}=0.01\right)$. Additionally, the normalized time $\mathrm{t}^{+}$ $\left(=\mathrm{t} \mathrm{U}_{\mathrm{b}} / \delta_{\mathrm{h}}\right)$ for averaging data was about 1750 (Peet and Sagaut (2009), Peet et al. (2010)).

(a)

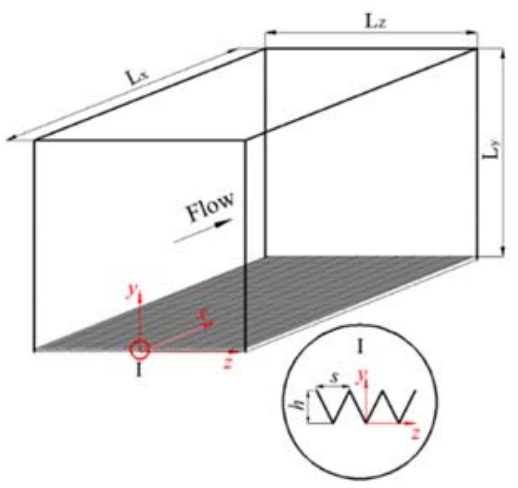

(b)

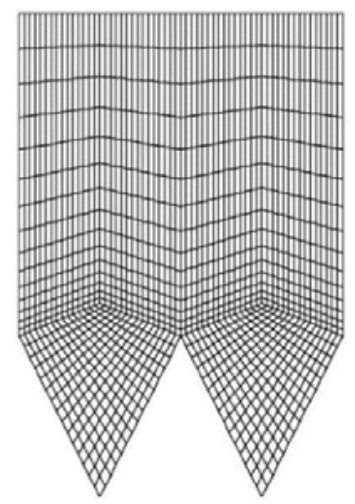

Fig. 3. CFD scheme: (a) sketch of computational domain; (b) streamwise slice near riblets.

Moreover, considering the fact that LES did not resolve but modeled the small-scale range $\left(\mathrm{h}^{+}=\mathrm{s}^{+}=15\right)$ over the riblet, the capture of the small-scale dynamics was extremely important. To verify this for the present computation, the premultiplied energy spectra was computed by conducting Fourier transform of fluctuating velocity data at $y^{1+}=4.4$. Based on this, $\lambda^{+}$lied in 50 3000, with a maximum energy at $300 \sim 1400$, while the scales in $\mathrm{y}$ and $\mathrm{z}$ direction, i.e., $\lambda^{+}$and $\lambda^{+}$, ranged from $1 \sim 500$, with a maximum energy accumulating at $8 \sim 140$. Besides, a rather fine grid resolution was adopted in present study. Specifically, the first grid point in y direction was kept at $\Delta y=0.53$ over the riblet surface and the $\mathrm{x}$ and z-grid spacing was kept at $\Delta \mathrm{x}^{+}=32$ and $\Delta \mathrm{z}^{+}=$ 0.5 , respectively. As a result, our CFD simulation could depict the minimum scales of 3D flow structures up to $\lambda^{+}=45, \lambda^{+}=1$ and $\lambda^{+}=1$.
Furthermore, these grid resolutions were much higher than some previous publications pursuing more re-fined flow structures (Peet et al. (2009), Bannier et al. (2016)). Evidently, our LES computation was capable of capturing the smallscale dynamics of the turbulent flow in the present near-wall range. As a typical example, a streamwise slice near riblets was indicated in Fig. 3(b), where the topological mesh structure could be observed. In addition, the validations of the numerical method were carried out by comparing the numerical data with the experimental ones, and the results were given in section 4 as these validated results were also used to study the effects of riblets on drag reduction and turbulent flows.

\subsection{Vortex Dynamic Scheme}

In order to acquire a deeper understanding on the mechanism of riblet drag reduction, the quantitative relationship between skin-friction and vorticity fields was also established from the view point of vortex dynamic theory, based on the data provided by the aforementioned CFD method. By doing so, it would be easier to analyze how the modified vortex structures affected the redistribution of local viscous drag force around the riblets.

Actually, for the sliced plane (S) at any spanwise location of the riblet [shown in Fig. 4], the line integral of skin-friction $\tau$ along the boundary of $S$ (ABCD) can be expressed using Eq. (1), following the Stokes-Theorem (Wu et al. (2015)),

$\oint_{\partial S} \boldsymbol{\tau} d \boldsymbol{l}=\int_{S}\left(\boldsymbol{n}_{\boldsymbol{s}} \times \nabla\right) . \boldsymbol{\tau} d S$

Here, the direction vector $\mathbf{n}_{\mathbf{s}}$ was normal to $\mathrm{S}$. $\mathrm{A}_{\mathrm{c}^{-}}$ cordingly, the streamwise skin-friction $\tau_{x}$ might be calculated using Eq. (2),

$\oint_{\partial S} \tau_{x} d x=\int_{S}-\frac{\partial \tau_{x}}{\partial y} d S$

Besides, according to $\mathrm{Wu}$ et al. (2007), the general relation between $\boldsymbol{\tau}$ and $\boldsymbol{\omega}$ was indicated as

$\boldsymbol{\tau}=\mu\left(\boldsymbol{\omega} \times \boldsymbol{n}_{\boldsymbol{r}}\right)$

Here, the direction vector $\mathbf{n}_{\mathbf{r}}$ was normal to the riblet side. Using Eq. (3), we could deduce

$\tau_{x}=-\mu \omega_{y} \sin \alpha-\mu \omega_{z} \cos \alpha$

Here, $\alpha$ was the base angle of the triangular riblet, as displayed in Fig. 4. By integrating Eq. (4) into (2), we got

$\oint_{\partial S} \tau_{x} d x=\int_{S}\left(\mu \frac{\partial \omega_{y}}{\partial y} \sin \alpha-\mu \frac{\partial \omega_{z}}{\partial y} \cos \alpha\right) d S$

Ideally, Eq. (5) could be further simplified into Eq. (6),

$\int_{l_{A B}} \tau_{x} d x \approx \int_{S}\left(\mu \frac{\partial \omega_{y}}{\partial y} \sin \alpha-\mu \frac{\partial \omega_{z}}{\partial y} \cos \alpha\right) d S$

Then the mean streamwise skin-friction, corresponding to the wall skin-friction at any spanwise S-plane, i.e., $\bar{\tau}_{r}=\bar{\tau}_{x}$, could be written as

$$
\bar{\tau}_{r} \approx \underbrace{\frac{\rho}{l_{A B}} \int_{S}\left(v \frac{\partial \bar{\omega}_{y}}{\partial y} \sin \alpha\right) d S}
$$


M. M. Zhang et al. / JAFM, Vol. 13, No. 4, pp. 1093-1106, 2020.

$$
\begin{gathered}
\bar{\tau}_{\mathrm{d} 1} \\
+\frac{\rho}{l_{A B}} \int_{S}\left(v \frac{\partial \bar{\omega}_{z}}{\partial y} \cos \alpha\right) d S \\
\bar{\tau}_{\mathrm{d} 2}
\end{gathered}
$$

Similarly, the skin-friction over smooth plate $\left(\tau_{\mathrm{s}}\right)$ could be denoted as

$\bar{\tau}_{S}=\frac{\rho}{l_{A B}} \int_{S}\left(v \frac{\partial \bar{\omega}_{z}}{\partial y}\right) d S$

$$
\bar{\tau}_{\mathrm{d} 2}
$$

The Eq. (8) was consistent with the findings of Eyink (2008). As Eyink mentioned, the generated vortices on the smooth wall were transported and diffused outward, forming the so-called spatial vorticity cascade. Considering the homogenous turbulent flow, Eyink further concluded that the normal diffusion flux of the dominant spanwise vortices, $\left(v \partial \bar{\omega}_{z} / \partial \mathrm{y}\right)$, which corresponded to the viscous energy dissipation, determined the magnitude of the viscous drag over the flat plate. Furthermore, Eyink's observation was also applicable to the riblet case. Obviously, $\bar{\tau}_{r}$ was composed of $\tau_{\mathrm{d} 1}$ and $\tau_{\mathrm{d} 2}$, whose magnitudes were dependent on the distributions of $v \partial \bar{\omega}_{y} / \partial \mathrm{y} \sin \alpha$ and $v \partial \bar{\omega}_{z} / \partial \mathrm{y} \cos \alpha$ within S-plane, respectively. Besides, $v \partial \bar{\omega}_{y} / \partial \mathrm{y} \sin \alpha$ and $v \partial \bar{\omega}_{z} / \partial \mathrm{y} \cos \alpha$ approximately corresponded to nor-mal diffusion flux of $\bar{\omega}_{y} \sin \alpha$ and $\bar{\omega}_{z} \cos \alpha$ (Wu et al.(2007), Eyink (2008), Adrian et al. (2000)), i.e., the decompositions of $\bar{\omega}_{y}$ and $\bar{\omega}_{z}$ over the riblet wall. The detailed results and discussions will be described in section 4 .

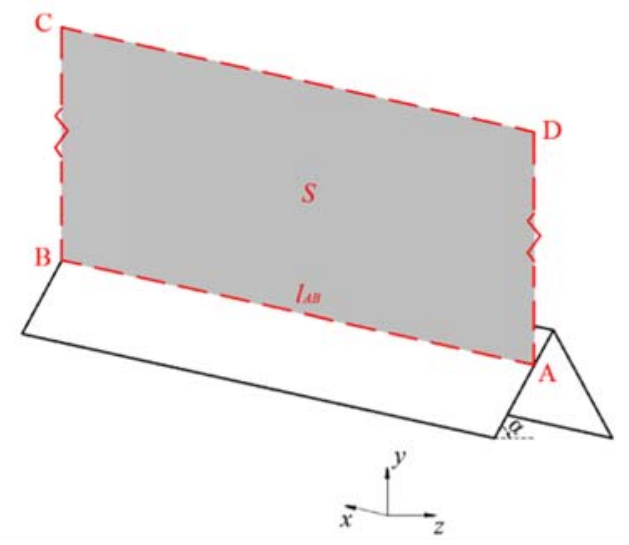

Fig. 4. Sketch of vortex dynamic analysis over triangular riblet.

\section{RESULTS AND DISCUSSIONS}

Figure 5 presented the dependence of the measured and computed drag reduction rate (DR) on the normalized spanwise location, i.e., $z / \mathrm{s}$. The magnitude of DR was calculated using the following equation,

$D R=\frac{\Delta \bar{\tau}}{\bar{\tau}_{s}} \times 100 \%=\frac{\bar{\tau}_{r}=\bar{\tau}_{s}}{\bar{\tau}_{s}} \times 100 \%$

Evidently, the experimental and numerical results were close to a certain extent. Moreover, the dashed line, corresponding to $\mathrm{DR}=0$, separated the zone between the rib valley and peak into two cases, i.e., the local drag decreasing and increasing cases, as seen in Fig. 5(a). The former changed gently, while the later varied sharply with a relatively smaller area, which led to the total DR of $6.23 \%$. In addition, it is also necessary to ensure that our LES method could give the proper prediction of the effect of riblet with different sizes. For this purpose, the LES computations have been conducted under three different riblet sizes, i.e., $\mathrm{s}^{+}=\mathrm{h}^{+}=10.1, \mathrm{~s}^{+}=\mathrm{h}^{+}=$ 24.7 and $\mathrm{s}^{+}=\mathrm{h}^{+}=26.9$. All these results, together with some typical experimental data from previous literature (Dean and Bhushan (2010), Walsh (1982), Bechert et al. (1987)), were shown in Fig. 5(b). Noted that the riblet size at $\mathrm{s}^{+}=10.1,15.8$ and 24.7 corresponded to the total drag decreasing cases, i.e., DR of $-4.59 \%,-6.23 \%$ and $-2.25 \%$, while the riblet size at $\mathrm{s}^{+}=26.9$ lied in the total drag increasing region, i.e., $\mathrm{DR}=1.84 \%$. Evidently, the "optimal drag reduction condition" at $\mathrm{s}^{+}=\mathrm{h}^{+}=15.8$ was directly proved.

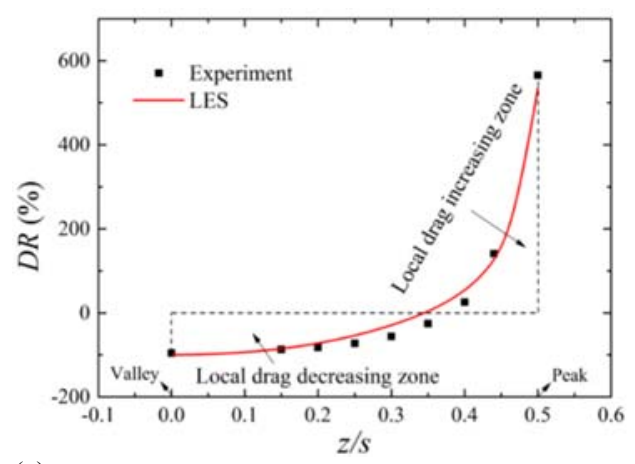

(a)

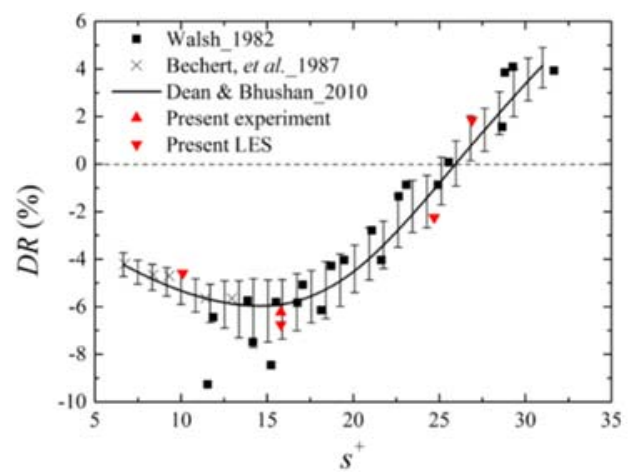

(b)

Fig. 5. The variation in the drag reduction rate (DR) with normalized spanwise location $(z / \mathrm{s})(\mathrm{a})$ and normalized spacing of riblet $\left(\mathrm{s}^{+}\right)(\mathrm{b})$.

The corresponding mean and fluctuating flow fields over the riblet groove were also studied by means of the experiment and computation. Figure 6 dis-played the typical normalized mean velocity contour $\left(\mathrm{U} / \mathrm{U}_{\infty}\right)$. Clearly, these two kinds of results agreed very well. In addition, the quasi-static fluid region quickly shrunken from $z / \mathrm{s}=0$ to 0.5 , suggesting that there was violent activity near the rib tip. This 
phenomenon was agreeable with the statement of the secondary vortex (Chu and Karniadakis (1993), Boomsma and Sotiropoulos (2016)). Furthermore, the effect of the riblet on the temporally and spatially averaged streamwise velocity U-profiles was shown in Fig. 7 by using numerical (curve line) and experimental (colored symbol) data. Spanwise positions at the rib valley $(z / \mathrm{s}=0)$ and peak $(z / \mathrm{s}=$ 0.5 ) were chosen to investigate the typical conditions within the local drag decreasing and increasing zones, respectively. Clearly, the computational results coincided well with the experimental ones. Compared with the smooth surface case, $U$ near the wall $\left(y^{\prime} / \mathrm{h}<2\right)$ was much smaller in the riblet valley and relatively larger in the riblet tip, implying the impaired and the enhanced momentum transport of mean flow field, respectively. Correspondingly, the representative profiles of turbulence intensity $(I)$ with relative coordinate $y^{\prime}$ to the local surface were indicated in Fig. 8. Again, the results of the numerical and experimental were consistent with each other. It was noticed that, irrespective of conditions, $I$ rapidly increased with the increasing of $y^{\prime}$, and above certain $y^{\prime}$, it tended to decrease and coincide at the end. The corresponding $y^{\prime}$ at the occurrence of maximum $I$ in the peak case was smaller than that in the valley case. In other words, the situation that the maximum $I$ happened at higher $y^{\prime}$ implied that the influence of the local drag decreasing case on the streaks and subsequent burst was relatively weaker than that of the local drag increasing one. These findings were consistent with the observations in Ei-Samni et al. (2005). For the smooth plate, a higher magnitude of maximum $I$ than its two counterparts was observed at a location $y^{\prime}$ between them, especially for the $I_{u}$ and $I_{v}$. Furthermore, the analogical trend of the Reynolds shear stress $\left(-\overline{u^{\prime} v^{\prime}} / \mathrm{U}_{\infty}{ }^{2}\right)$ with $y^{\prime} / \mathrm{h}$ was also shown in Fig. 9, explaining the variation of skin-friction from another aspect. In addition, the results in Figs. 6-9 validated our LES method which would be applied to conduct the following investigations.

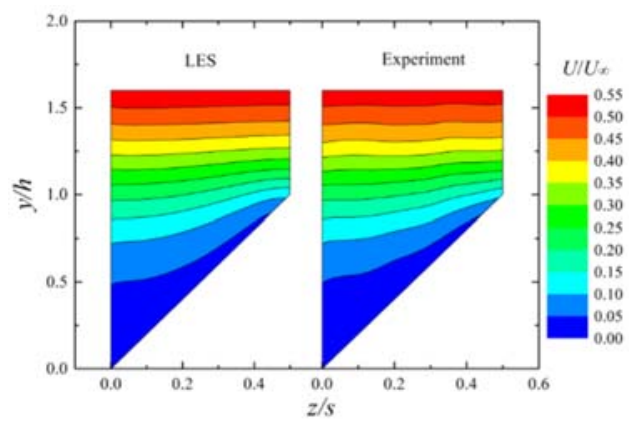

Fig. 6. Typical comparisons between experimental and numerical mean velocity contours over riblet groove.

Next, the deduced vortex dynamic model in section 3.2 (Eq. 7) was utilized to further interpret the flow physics behind the effect of the riblets, as this equation established the relation between the local wall skin-friction and the vortex flows over the riblet surface. For this purpose, besides the riblet peak $(z$
$/ \mathrm{s}=0.5$ ), another two conditions at $z / \mathrm{s}=0.2$ and 0.3 over the riblet groove, representing intermediate states for the local drag decreasing zone, were also chosen as research subjects. Figure 10 indicated five representative instantaneous fluctuating $\tau_{\mathrm{r}}$ along the whole riblet test plate at the above three spanwise locations. The smooth case was also presented for comparison. It should be noted that $\tau_{\mathrm{r}}$ at the valley $(z$ $/ \mathrm{s}=0)$ was quite small and thus was not given here. As expected, $\tau_{\mathrm{r}}$ was evidently impaired at $z / \mathrm{s}=0.2$ and 0.3 and enhanced at $z / \mathrm{s}=0.5$, compared with $\tau_{\mathrm{s}}$. Moreover, irrespective of the span-wise location, $\tau_{\mathrm{r}}$ varied intermittently and tended to be similar in the streamwise direction at the same instant [Figs. 10(b)(d)]. This was possibly related to the effect of the 'burst' phenomenon of coherent structures which had a much larger scale than that of riblet spacing $\left(\mathrm{s}^{+}\right.$ $=15.8$ ) (Adrian (2007)). In or-der to detect the burst events in turbulent boundary layers, by using the VITA (variable-interval time averaging) method (Chu and Karniadakis (1993), Ghanadi et al. (2014)), the normalized bursting frequencies $\mathrm{f}_{\mathrm{b}}{ }^{+}$, i.e., $\mathrm{f}_{\mathrm{b}}{ }^{+}=$ $\mathrm{f}_{\mathrm{b}} \delta_{\mathrm{h}} / \mathrm{U}_{\mathrm{b}}$, of the coherent structures over the smooth and riblet surfaces, were about 0.182 and 0.152 , respectively (the bursting frequencies were calculated at the location of $\mathrm{y}^{+}=20$ (Vinuesa et al. (2015))). This suggested that the bursting events in the outer layer were impeded by riblets, coinciding with some previous findings (Viswanath (2002), Choi (1989), Chu and Karniadakis (1993)).

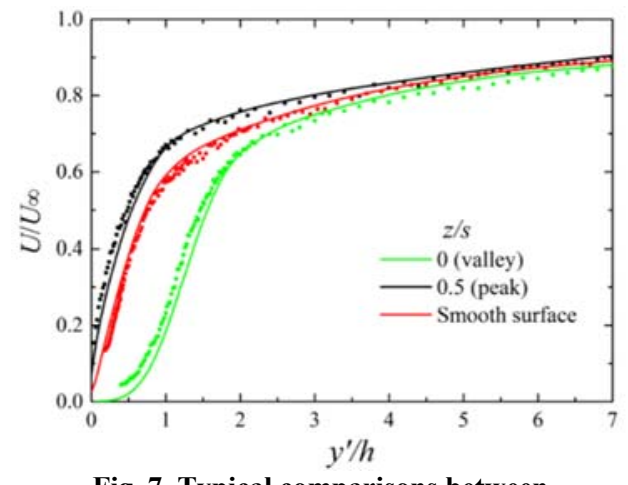

Fig. 7. Typical comparisons between experimental and numerical mean velocity profiles at different spanwise locations.

The spanwise variations of the mean skin-friction $\bar{\tau}_{\mathrm{r}}$ $\left(=\bar{\tau}_{\mathrm{d} 1}+\bar{\tau}_{\mathrm{d} 2}\right)$ and its decompositions $\left(\bar{\tau}_{\mathrm{d} 1}\right.$ and $\left.\bar{\tau}_{\mathrm{d} 2}\right)$ over the riblet surface were illustrated in Fig. 11, which was obtained using LES computation. In order to make this decomposition more significant to understand the drag reduction mechanism, a total drag increasing case, i.e., $\mathrm{s}^{+}=26.9$ with $\mathrm{DR}=1.84 \%$, was added to compare with the total drag decreasing case $\left(\mathrm{s}^{+}=15.8\right.$ with $\left.\mathrm{DR}=-6.23 \%\right)$. All these quantities were calculated using Eqs. (7) and (8), and curve-fitted at different $z / \mathrm{s}$. For the total drag decreasing case $\left(\mathrm{s}^{+}=15.8\right.$, solid lines), clearly, the general trend of $\tau_{\mathrm{r}}$ was consistent with Fig. 5(a). Moreover, $\tau \mathrm{d} 1$ was successively enhanced and was superior over $\bar{\tau}_{\mathrm{d} 2}$ until $z / \mathrm{s} \approx 0.4$. After that, $\bar{\tau}_{\mathrm{d} 2}$ increased significantly all the way to the riblet tip 
M. M. Zhang et al. / JAFM, Vol. 13, No. 4, pp. 1093-1106, 2020.

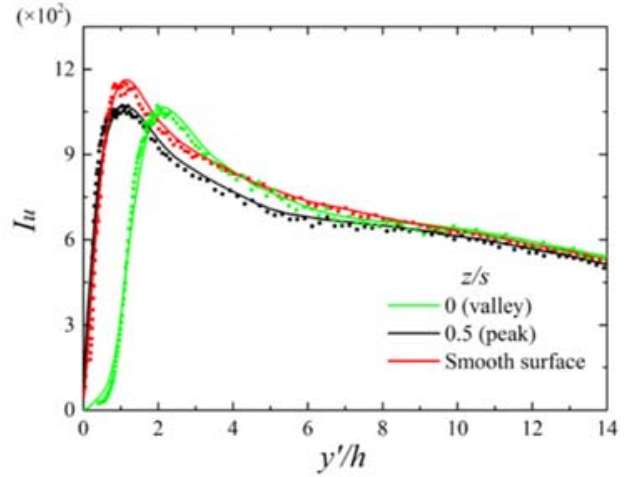

(a)

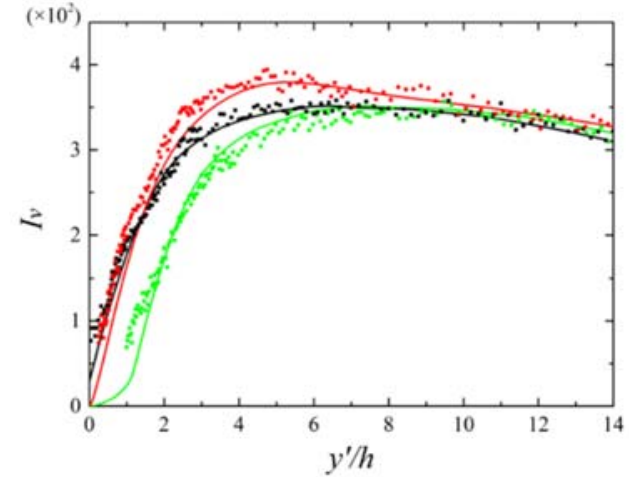

(b)

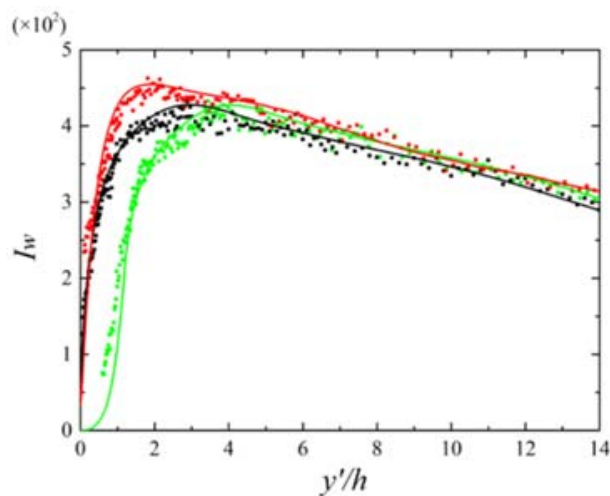

(c)

Fig. 8. Typical comparisons between experimental and numerical turbulence intensity profiles at different spanwise locations: (a) $I_{u}$; (b) $I_{v} ;$ (c) $I_{w}$.

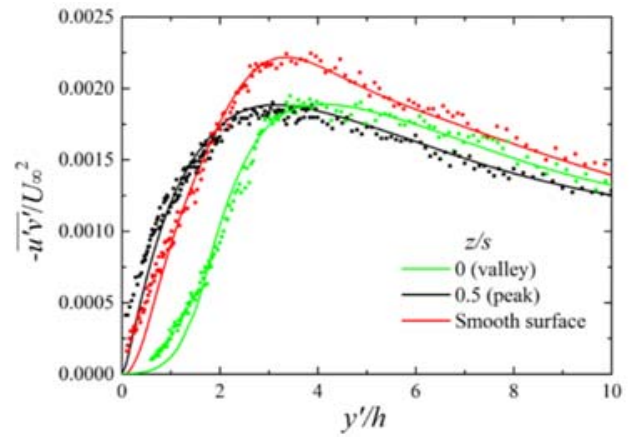

Fig. 9. Typical comparisons between experimen-tal and numerical Reynolds shear stress $\left(-\overline{u^{\prime} v^{\prime}} / \mathbf{U}_{\infty}{ }^{2}\right)$ profiles at different spanwise locations.

$(z / \mathrm{s}=0.5)$ and $\bar{\tau}_{\mathrm{d} 1}$ decreased rapidly. From another perspective, around the riblet tip area, the sum of the normal diffusion flux of $\bar{\omega}_{\mathrm{z}} \cos \alpha$ over the riblet wall contributed much more to $\bar{\tau}_{\mathrm{r}}$, while within other regions, the local drag decreasing zone in particular, the sum of the normal diffusion flux of $\bar{\omega}_{\mathrm{y}} \sin \alpha$ over the riblet wall mainly determined the magnitude of $\bar{\tau}_{\mathrm{r}}$. As for the total drag increasing case $\left(\mathrm{s}^{+}=26.9\right.$, dashed lines), the decomposition of the skin-friction still worked and the general trends of $\bar{\tau}_{\mathrm{r}}, \bar{\tau}_{\mathrm{d} 1}$ and $\bar{\tau}_{\mathrm{d} 2}$ were similar with those for the total drag deceasing case. However, the magnitudes were different. In the local drag increasing zone, these three quantities increased, while they were unchanged in most part of the local drag decreasing zone. Thus, a wider local drag increasing zone and a smaller local decreasing zone were observed, leading to the increasing of the total skin-friction. Besides, given the fact that the riblet spacing $\left(\mathrm{s}^{+}=26.9\right)$ for the total drag increasing case was larger than that $\left(\mathrm{s}^{+}=15.8\right)$ for the total drag decreasing case, it was reasonable to say that more vortical scales were penetrated into the riblet grooves, leading to more intense vortical motions near the riblet peak region. Further analysis revealed that, from the total drag decreasing case to the total drag increasing case, other related vortical quantities 


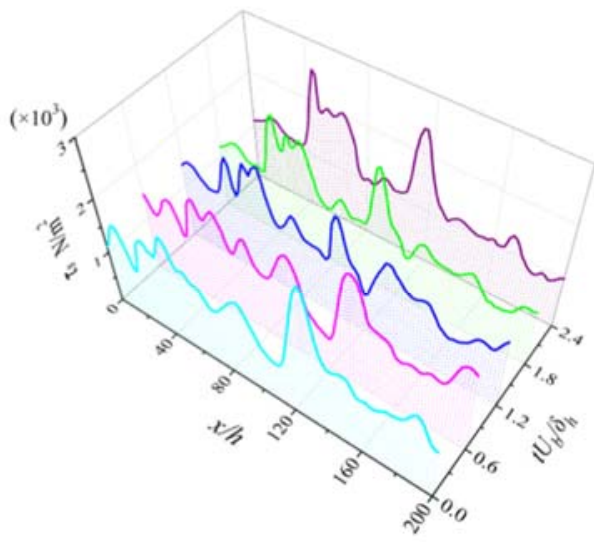

(a) Smooth surface

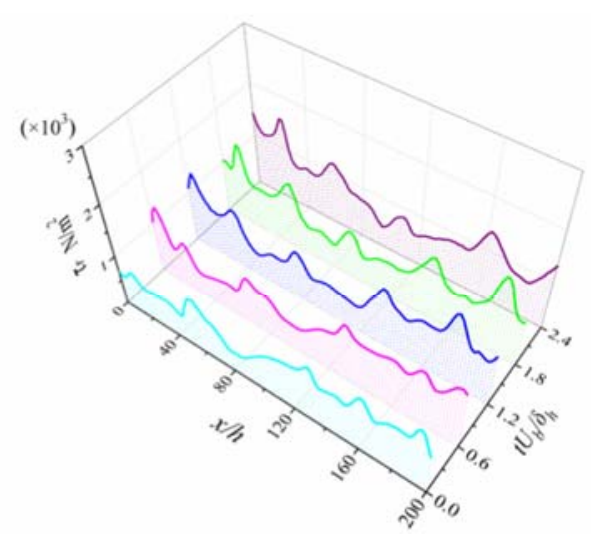

(c) $z / s=0.3$ (riblet surface)

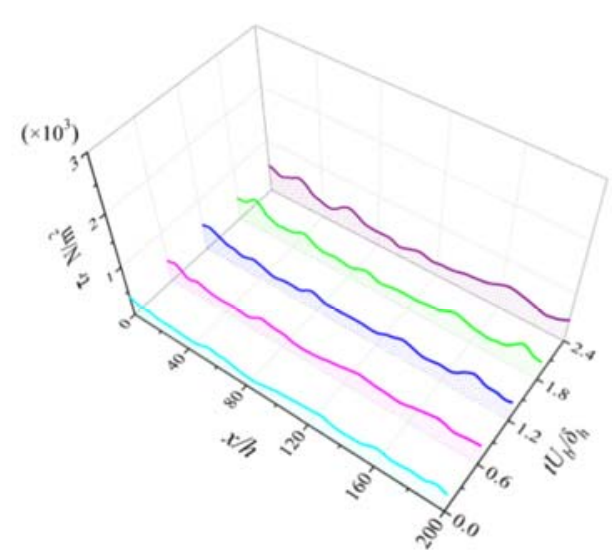

(b) $z / s=0.2$ (riblet surface)

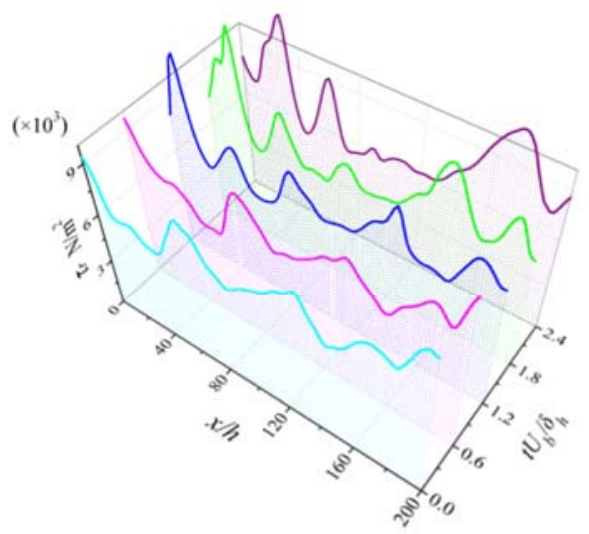

(d) $z / s=0.5$ (riblet surface)

Fig. 10. Typical instantaneous streamwise skin-friction over smooth and riblet surfaces at different spanwise locations.

discussed in the following part also had a similar variation with $\bar{\tau}_{\mathrm{r}}, \bar{\tau}_{\mathrm{d} 1}$ and $\bar{\tau}_{\mathrm{d} 2}$. In order to avoid the repetitive descriptions and meanwhile, put more emphasis on the "optimal drag reduction condition", the following section would discuss results for the total drag decreasing case $\left(\mathrm{s}^{+}=15.8\right)$.

The variation of the vortex transportation in Fig. 11 must be closely related to the dynamics of the vortical flows. To support this, the time-averaged $z$ vorticity $\left(\bar{\omega}_{z}\right)$ over the smooth plate, and y-vorticity $\left(\bar{\omega}_{\mathrm{y}}\right)$ and $\bar{\omega}_{\mathrm{z}}$ over the riblet plate, were individually computed in Fig. 12. Noted $\bar{\omega}_{\text {z }}$ predominated on the smooth surface [Fig. 12(a)] and thus determined the value of $\bar{\tau}_{\mathrm{s}}$ according to Eq. (8). Besides, com-pared with the smooth surface, the normal vorticity $\left(\bar{\omega}_{\mathrm{y}}\right)$ dominated most of the local drag decreasing and part of the increasing zones, but with less strength [Fig. 12(b)]. In contrast, the mean spanwise vorticity $\left(\bar{\omega}_{z}\right)$, with a higher strength, was remarkably near the riblet peak region, as displayed in Fig. 12(c).

In addition, the specific aggregations of $\bar{\omega}_{\mathrm{y}}$ and $\bar{\omega}_{\mathrm{z}}$ could be further understood by computing the corresponding boundary vortex flux $(\mathbf{B V F}, \boldsymbol{\sigma})$. With a minus sign denoting the generation and viscous diffusion flux of vorticity near the wall, the vector $\sigma$ was defined by Eq. (10) (Wu et al. (2015)):

$\boldsymbol{\sigma}=-v \frac{\partial \omega}{\partial n_{r}}$

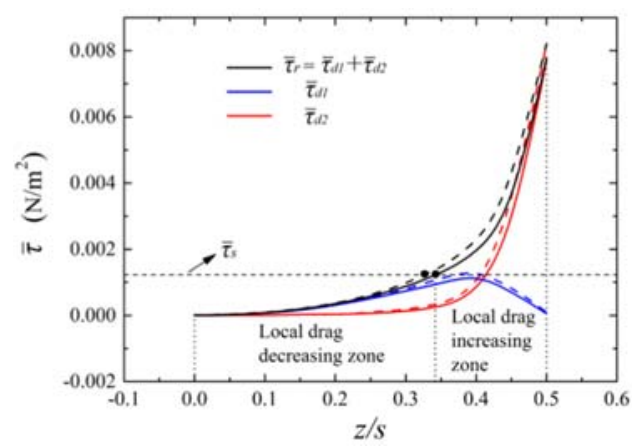

Fig. 11. Spanwise variation of mean skin-friction $\overline{\boldsymbol{\tau}}_{\mathrm{r}}\left(=\overline{\boldsymbol{\tau}}_{\mathrm{d} 1}+\overline{\boldsymbol{\tau}}_{\mathrm{d} 2}\right)$ based on LES computation. Solid lines: total drag decreasing case $\left(\mathrm{s}^{+}=15.8\right)$, dashed lines: total drag increasing case $\left(\mathrm{s}^{+}=\right.$ 26.9). Black dots: cut point between the local drag de-creasing zone and the local drag increasing zone. 


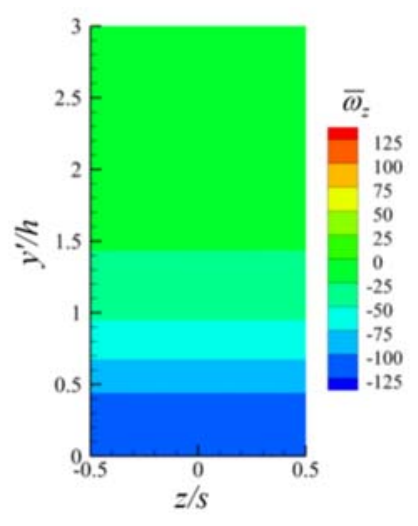

(a) $\bar{\omega}_{z}$ (smooth)

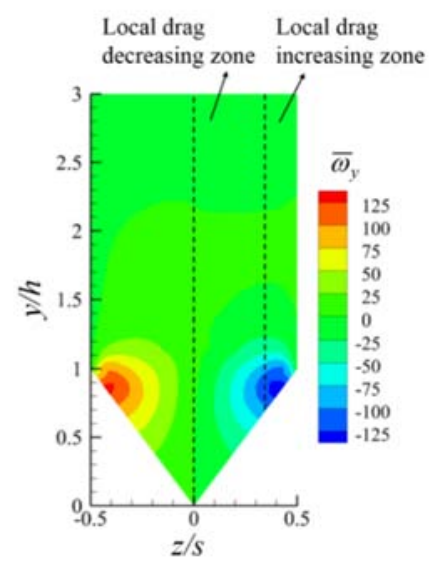

(b) $\bar{\omega}_{y}$ (riblet)

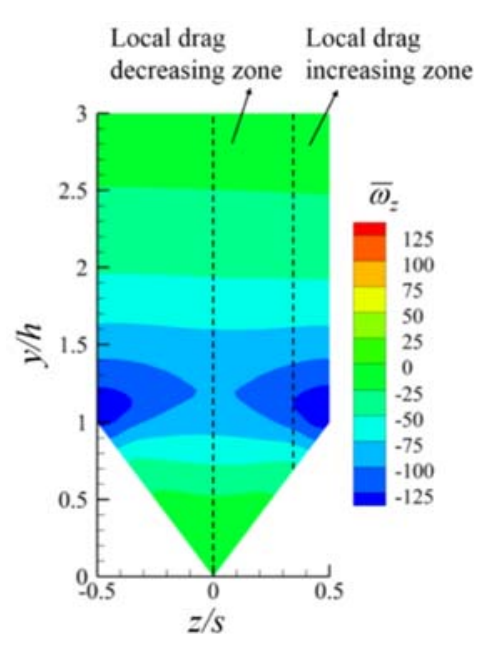

(c) $\bar{\omega}_{z}$ (riblet)

Fig. 12. Time-averaged vorticities over smooth and riblet plates.

According to Eq. (10), the BVF in $y$ and $z$ directions ( $\sigma_{y}$ and $\sigma_{z}$ ) could be obtained using Eqs. (11) and (12),

$\sigma_{y}=-v \frac{\partial \omega_{y}}{\partial n_{r}}$ $\sigma_{z}=-v \frac{\partial \omega_{z}}{\partial n_{r}}$

The typical time-averaged distributions $\left(\sigma_{y}\right.$ and $\sigma_{z}$ ) along the riblet side were shown in Fig. 13. The minus $\sigma_{y}$ and $\sigma_{z}$ separately appeared in the range of $z / \mathrm{s}=0.1-0.4$ and $z / \mathrm{s}=0.4-0.5$, where normal and spanwise vortices occupied the corresponding regions, in line with the findings in Figs. 11 and 12.

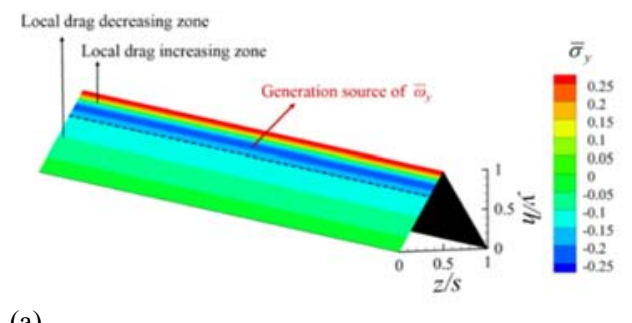

(a)

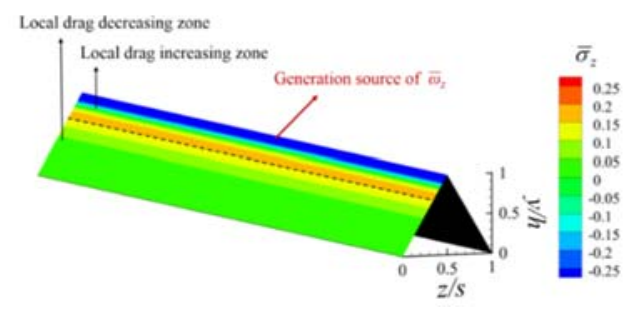

(b)

Fig. 13. Time-averaged distributions of boundary vortex fluxes $\left(\overline{\boldsymbol{\sigma}}_{y}\right.$ and $\left.\overline{\boldsymbol{\sigma}}_{z}\right)$ on the riblet wall.

Furthermore, the way these generated vortices $\left(\bar{\omega}_{y}\right.$ and $\bar{\omega}_{z}$ ) diffused into the outer flow over the riblet groove was also interesting, since the sum of the normal diffusion fluxes, i.e., $v \partial \bar{\omega}_{y} / \partial y \sin \alpha$ and $v \partial \bar{\omega}_{z} / \partial y \cos \alpha$, determined the magnitudes of $\bar{\tau}_{\mathrm{d} 1}$ and $\bar{\tau}_{\mathrm{d} 2}$ according to Eq. (7). The results were revealed in Fig. 14 and the one for the smooth case was also displayed for reference. From the mean contour $v \partial \bar{\omega}_{y} / \partial y \sin \alpha$ in Fig. 14(b), both the contour value and scale were notably enlarged in the local drag decreasing zone, and they were even larger within the partial region for the local drag increasing zone, corresponding to the enhanced $\bar{\tau}_{\mathrm{d} 1}$. Nevertheless, the magnitude of $v \partial \bar{\omega}_{y} / \partial \mathrm{y} \sin \alpha$ near the riblet tip totally vanished, signifying the fact that there was a sudden shrink of $\tau \mathrm{d} 1$. For the same to-ken, a rather wide area of $v \partial \bar{\omega}_{z} / \partial y \cos \alpha$ with only positive sign prevailed overwhelmingly near the riblet peak, hinting that there was a rapid increasing of $\bar{\tau}_{\mathrm{d} 2}$. In contrast to the mean contour $v \partial \bar{\omega}_{z} / \partial y$ for the smooth case, the two contour values of $v \partial \bar{\omega}_{y} / \partial y \sin \alpha$ and $v \partial \bar{\omega}_{z} / \partial y \cos \alpha$ over the riblet groove were evidently smaller and larger within the local drag decreasing and increasing zones, respectively, resulting in a lower $\bar{\tau}_{\text {r }}$ around the riblet valley and higher $\bar{\tau}_{\mathrm{r}}$ around the riblet peak.

From another point of view, as $\bar{\omega}_{y}=\partial \mathrm{U} / \partial z$ and $\bar{\omega}_{z}=$ $-\partial \mathrm{U} / \partial \mathrm{y}$, we got

$v \frac{\partial \bar{\omega}_{y}}{\partial y} \sin \alpha+v \frac{\partial \bar{\omega}_{z}}{\partial y} \cos \alpha=-v \frac{\partial^{2} U}{\partial y \partial n_{r}}$ 


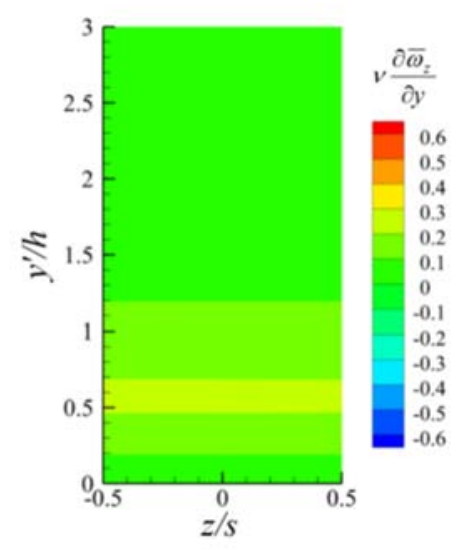

(a) $v \frac{\partial \bar{\omega}_{z}}{\partial y}$ (smooth)

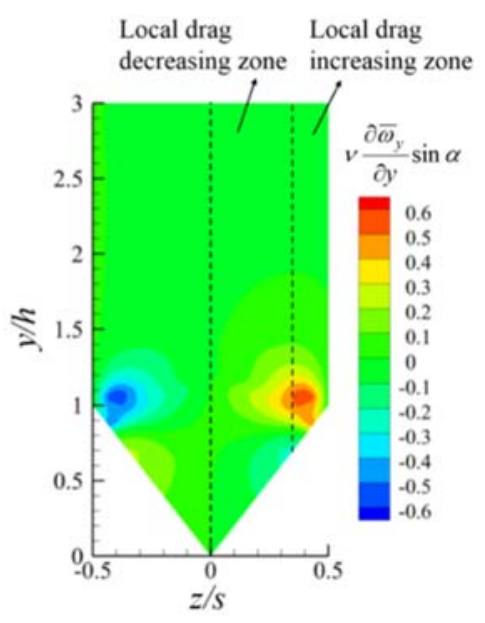

(b) $v \frac{\partial \bar{\omega}_{y}}{\partial y} \sin \alpha$ (riblet)

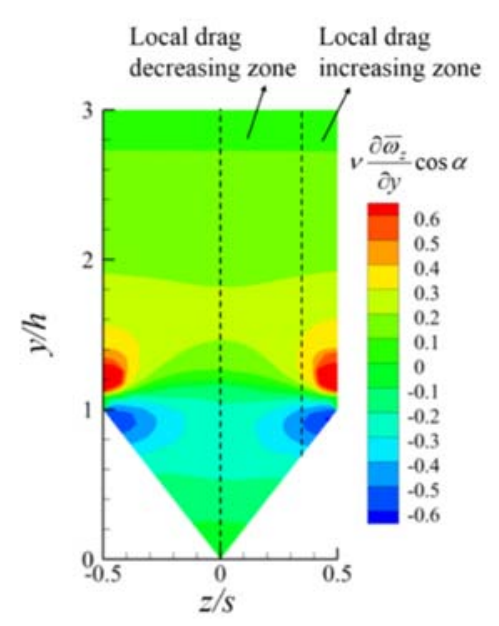

(c) $v \frac{\partial \bar{\omega}_{y}}{\partial z} \cos \alpha$ (riblet)

Fig. 14. Time-averaged contours of normal diffusion flux over smooth and riblet plates.

Thus, the magnitude of $\bar{\tau}_{\text {r }}$ was determined by the distribution of $-v \partial^{2} U /\left(\partial y \partial \mathrm{n}_{\mathrm{r}}\right)$ in the flow field. Similarly, the magnitude of $\bar{\tau}_{\text {s }}$ was determined by the distribution of $-v \partial^{2} U / \partial y^{2}$. In order to clarify the physical meaning of $-v \partial^{2} \mathrm{U} / \partial \mathrm{y}^{2}$ for the smooth case and $-v \partial^{2} U /\left(\partial y \partial \mathrm{n}_{\mathrm{r}}\right)$ for the riblet case, the incompressible Navier-Stokes equation in the $\mathrm{x}$ direction was given as

$$
\underbrace{\frac{\partial u}{\partial t}}+\underbrace{u \frac{\partial u}{\partial x}+v \frac{\partial u}{\partial y}+w \frac{\partial u}{\partial z}}=-\frac{1}{\rho} \frac{\partial p}{\partial x}
$$

Unsteady term convective term pressure term

$$
\underbrace{+v \nabla^{2} u}_{\text {Viscous term }}
$$

In the time-averaged flow field, the viscous diffusion term for the smooth case could be denoted as $v \nabla^{2} u=$ $v \partial^{2} \mathrm{U} / \partial \mathrm{y}^{2}=-v \partial \bar{\omega}_{z} / \partial \mathrm{y}$, which meant that $-v \partial \bar{\omega}_{z} / \partial \mathrm{y}$ $\left(v \partial^{2} \mathrm{U} / \partial \mathrm{y}^{2}\right)$ corresponded to the viscous diffusion effect in the turbulent boundary layer. Furthermore, this finding was also applicable in the riblet case where $v \partial \bar{\omega}_{y} / \partial y \sin \alpha$ and $v \partial \bar{\omega}_{z} / \partial y \cos \alpha$ represented two components of the viscous term.

In turbulent flows, the viscous diffusion had a significant effect on the near-wall coherent motions and the turbulent energy transportation (Wu et al. (2007), Eyink (2008)). By using Q-criterion, the near-wall turbulent streaks were indicated in Fig. 15. These coherent streaks made up a stress-producing cycle that led to the large peak in the turbulence production. Obviously, riblets had a notice-able effect on the distribution of turbulent streaks. Specifically, the scale of the streaks over the riblet surface was much smaller than that over the smooth surface. Besides, most of these smaller turbulent streaks clustered around the peak region, while few existed around the valley region. These observations could be seen that riblets disassembled and redistributed the near-wall turbulent streaks.

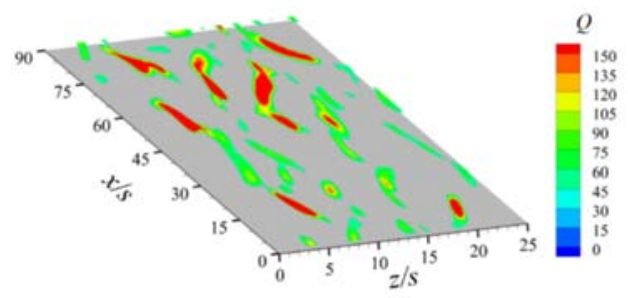

(a) Smooth surface

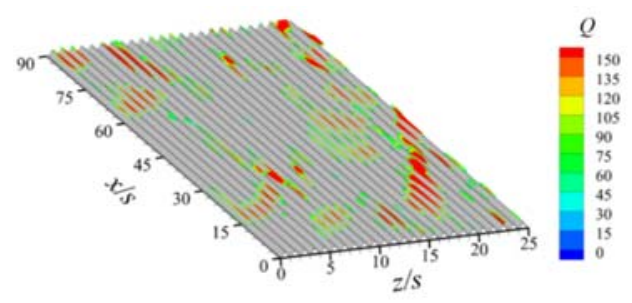

(b) Riblet surface

Fig. 15. Turbulent streaks over the smooth and riblet surfaces by using Q-criterion. 


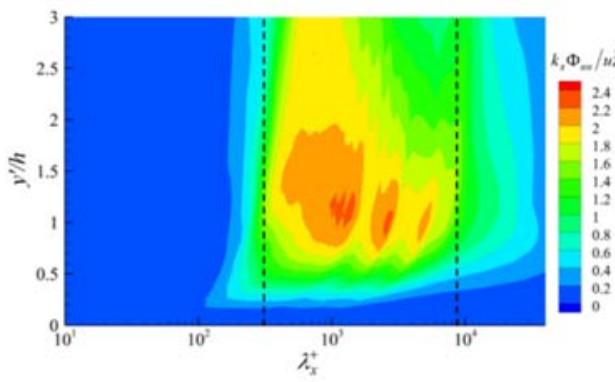

(a) Smooth surface

(a)

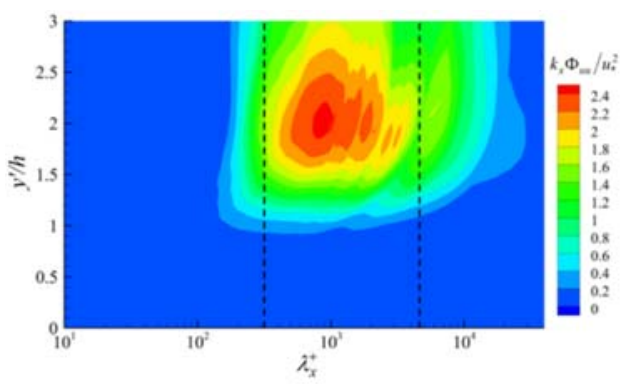

(b) $z / s=0$ (riblet valley)

(b)

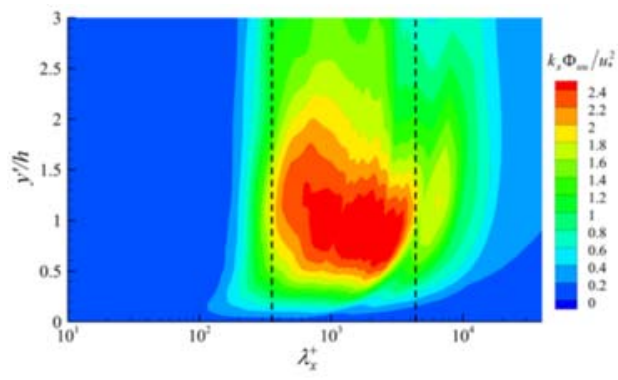

(c) $z / s=0.5$ (riblet peak)

(c)

Fig. 16. Pre-multiplied energy spectra of the streamwise velocity fluctuations, $k_{x} \varphi_{u u} / u^{2} *^{2}$, as a function of the wall-normal height and streamwise length scale.

The disassembled turbulent streaks would undoubtedly disturb the distribution of turbulent energy and thereafter influence the bursting process in the near-wall viscous region (Tang et al. (2016), Hutchins and Marusic (2007), Bentley (2004)). Therefore, the pre-multiplied energy spectra, same as the one described in section 3.1, was analyzed. The normalized energy spectra was given by $\mathrm{k}_{\mathrm{x}} \varphi_{\mathrm{uu}} / \mathrm{u} *^{2}$, where the streamwise wavenumber $\mathrm{k}_{\mathrm{x}}$ was calculated from the frequency $f$ and local mean velocity $U$, i.e., $\mathrm{k}_{\mathrm{x}}=2 \pi \mathrm{f} / \mathrm{U}$, and the energy of streamwise fluctuating velocities $\left(\varphi_{\text {uu }}\right)$ was calculated using

$\varphi_{u u}\left(k_{x}, y\right)=<{\widehat{u^{\prime}}}\left(k_{x}, y\right){\widehat{u^{\prime}}}^{*}\left(k_{x}, y\right)>$,

where $<>$ denoted the spatial and temporal average, $\widehat{u}^{\prime}$ was the Fourier transform of $u^{\prime}$ and $\widehat{u}^{\prime}{ }^{*}$ was the complex conjugate of $\widehat{u^{\prime}}$ (Chin et al. (2009)).

Figure 16 presented the pre-multiplied energy spectra plotted against the wall-normal distance and streamwise length scale $\left(\lambda_{\mathrm{x}}=1 / \mathrm{k}_{\mathrm{x}}\right)$ over the smooth and riblet surfaces. Roughly $70 \% \sim 80 \%$ of the turbulent energy was concentrated between two vertical dashed lines. Clearly, the energyconcentrated range for the smooth surface $\left(\lambda_{x}=310\right.$ $\sim 8500)$ was wider than that for the riblet valley and peak (valley: $\lambda_{\mathrm{x}}=320 \sim 4500$, peak: $\lambda_{\mathrm{x}}=350 \sim$ 4200). This confirmed the fact that the formation of turbulent streaks were impeded by riblets. In the riblet peak [Fig. 16(c)], due to the intense motions of turbulent streaks, the energy concentrated in the near-wall region $\left(y^{\prime} / \mathrm{h}=0.5 \sim 2\right)$ and was much larger than that in the smooth case. Since most of $\bar{\omega}_{y}$ and $\bar{\omega}_{z}$ generated around the riblet peak [indicated in Fig. 13], the increasing energy in the riblet peak meant that there was a strong viscous energy diffusion, i.e., $-v \frac{\partial^{2} U}{\partial y \partial n_{r}}\left(v \partial \bar{\omega}_{y} / \partial y \sin \alpha\right.$ and $v \partial \bar{\omega}_{z}$ $/ \partial \mathrm{y} \cos \alpha)$. In the riblet valley, although the turbulent energy was still larger than the one in the smooth case, the wall-normal height was much higher $\left(y^{\prime} / \mathrm{h}\right.$ $=1.5 \sim 3)$. This meant that vortical motions had little influence on the near-wall region. Due to the vanishing generation of $\bar{\omega}_{y}$ and $\bar{\omega}_{z}$ in the valley, the viscous diffusion ( $v \partial \bar{\omega}_{y} / \partial y \sin \alpha$ and $\left.v \partial \bar{\omega}_{z} / \partial y \cos \alpha\right)$ was relatively small. These findings also supported the facts that the impaired and enhanced flow fluctuation and thus $\bar{\tau}_{\mathrm{r}}$ existed in the local drag decreasing zone and the local drag in-creasing zone, as indicated in Figs. 7-10.

\section{CONCLUSIONS}

Using experimental and numerical methods, together with vortex dynamic analysis, the drag reduction mechanism of triangular riblets has been revisited by analyzing the generation and evolution of vortices induced by riblets. The results in this research could lead to the following conclusions:

1. The relation between the local spanwise skinfriction $\left(\bar{\tau}_{\mathrm{r}}\right)$ over riblet grooves and the vortical flows was built using the vortex dynamic method. The local spanwise skin-friction $\bar{\tau}_{\mathrm{r}}$ encompassed two terms: $\bar{\tau}_{\mathrm{d} 1}$ and $\bar{\tau}_{\mathrm{d} 2}$, which represented the effects of mean normal diffusion fluxes of normal and spanwise vorticities, respectively.

2. For the local drag decreasing zone, the magnitude of $\bar{\tau} \mathrm{d} 1$ was dependent on the distribution of the normal diffusion flux of $\bar{\omega}_{y}$ $\sin \alpha$ over the riblet wall. With relatively smaller vortex strength and viscous diffusion in this local zone, the turbulent energy in the near-wall region was attenuated, resulting in less averaged $\bar{\tau}_{\text {r than }} \bar{\tau}_{\mathrm{s}}$

3. For the local drag increasing zone, a much larger spanwise vortex strength generated near the riblet tip and thereafter diffused into the outer flow due to a stronger normal diffusion flux of $\bar{\omega}_{z} \cos \alpha$. Correspondingly, the turbulent energy increased, which finally led to a higher $\bar{\tau}_{\mathrm{d} 2}$ subsequent $\bar{\tau}_{\mathrm{r}}$ than $\bar{\tau}_{\mathrm{s}}$ over the flat plate. 
The content presented in this paper is only the results of the very first stage of our research, and in the future we will focus on the flow physics of the

riblets with different cross-sections, aiming to acquire a general prediction model for the engineering application.

\section{ACKNOWLEDGMENTS}

This work was supported by the National Natural Science Foundation of China (Grant No. 51736008), "Transformational Technologies for Clean Energy and Demonstration", Strategic Priority Research Program of the Chinese Academy of Sciences (Grant No. XDA21050303). Dr. S.F. Zou at Peking University was highly appreciated for his useful suggestions.

\section{REFERENCES}

Adrian, R. J. (2007). Hairpin vortex organization in wall turbulence. Physics of Fluids 19(4), 041301 .

Adrian, R. J., C. D. Meinhart, and C. D. Tomkins (2000). Vortex organization in the outer region of the turbulent boundary layer. Journal of Fluid Mechanics 422, 1-54.

Bacher, E. and C. R. Smith (1986). Turbulent boundary-layer modification by surface riblets. AIAA journal 24(8), 1382-1385.

Bannier, A., E. Garnier, and P. Sagaut (2016). Riblets induced drag reduction on a spatially developing turbulent boundary layer. In Progress in Wall Turbulence 2, 213-224. Springer.

Bechert, D. and M. Bartenwerfer (1989). The viscous flow on surfaces with longitudinal ribs. Journal of fluid mechanics 206, 105-129.

Bechert, D., D. Gerich, and G. Hoppe (1987). Short report on measurements with sawtooth riblets (3m plastic riblet film). DFVLR/HFI Berlin.

Bechert, D., M. Bruse, W. v. Hage, J. T. Van der Hoeven, and G. Hoppe (1997). Experiments on drag-reducing surfaces and their optimization with an adjustable geometry. Journal of fluid mechanics 338, 59-87.

Benschop, H. and W.-P. Breugem (2017). Drag reduction by herringbone riblet texture in direct numerical simulations of turbulent channel flow. Journal of Turbulence 18(8), 717-759.

Bentley, R. (2004). Uncertainty in measurement: the iso guide.

Boomsma, A. and F. Sotiropoulos (2016). Direct numerical simulation of sharkskin denticles in turbulent channel flow. Physics of Fluids 28(3), 035106 .

Chen, H., X. Zhang, L. Ma, D. Che, D. Zhang, and T. Sudarshan (2014). Investigation on large-area fabrication of vivid shark skin with superior surface functions. Applied Surface Science 316, 124-131.

Chin, C., N. Hutchins, A. Ooi, and I. Marusic (2009). Use of direct numerical simulation (dns) data to investigate spatial resolution issues in measurements of wall-bounded turbulence. Measurement Science and Technology 20(11), 115401.

Choi, H., P. Moin, and J. Kim (1993). Direct numerical simulation of turbulent flow over riblets. Journal of fluid mechanics 255, 503539.

Choi, K. S. (1989). Near-wall structure of a turbulent boundary layer with riblets. Journal of fluid mechanics 208, 417-458.

Chu, D. C. and G. E. Karniadakis (1993). A direct numerical simulation of laminar and turbulent flow over riblet-mounted surfaces. Journal of Fluid Mechanics 250, 1-42.

Dean, B. and B. Bhushan (2010). Shark-skin surfaces for fluid-drag reduction in turbulent flow: a review. Philosophical Transactions of the Royal Society A: Mathematical, Physical and Engineering Sciences 368(1929), 4775-4806.

Ei-Samni, O., H. S. Yoon, and H. H. Chun (2005). Turbulent flow over thin rectangular riblets. Journal of mechanical science and technology 19(9), 1801-1810.

Eyink, G. L. (2008). Turbulent flow in pipes and channels as cross-stream "inverse cascades" of vorticity. Physics of Fluids 20(12),125101.

García-Mayoral, R. and J. Jiménez (2011). Drag reduction by riblets. Philosophical Transactions of the Royal Society A: Mathematical, Physical and Engineering Sciences 369(1940), 1412-1427.

Ghanadi, F., M. Arjomandi, B. Cazzolato, and A. Zander (2014). Experimental investigation of the application of a self-excited cylindrical helmholtz resonator for turbulent drag reduction. In 19th Australasian Fluid Mechanics Conference.

Grüneberger, R. and W. Hage (2011). Drag char acteristics of longitudinal and transverse riblets at low dimensionless spacings. Experiments in Fluids 50(2), 363-373.

Heidarian, A., H. Ghassemi, and P. Liu (2018). Numerical analysis of the effects of riblets on drag reduction of a flat plate. Journal of Applied Fluid Mechanics 11(3), 679-688.

Huang, C., D. Liu, and J. Wei (2016). Experimental study on drag reduction performance of surfactant flow in longitudinal grooved channels. Chemical Engineering Science 152, 267-279.

Hutchins, N. and I. Marusic (2007). Large-scale influences in near-wall turbulence. Philosophical Transactions of the Royal Society A: Mathematical, Physical and Engineering 
M. M. Zhang et al. / JAFM, Vol. 13, No. 4, pp. 1093-1106, 2020.

Sciences 365(1852), 647-664.

Jiménez, J. and P. Moin (1991). The minimal flow unit in near-wall turbulence. Journal of Fluid Mechanics 225, 213-240.

Lee, S. J. and S. H. Lee (2001). Flow field analysis of a turbulent boundary layer over a riblet surface. Experiments in Fluids 30(2), 153-166.

Lee, S. J. and Y.-S. Choi (2008). Decrement of spanwise vortices by a drag-reducing riblet surface. Journal of Turbulence (9), N23.

Luchini, P., F. Manzo, and A. Pozzi (1991). Resistance of a grooved surface to parallel flow and cross-flow. Journal of Fluid Mechanics 228, 87-109.

Luo, Y., D. Zhang, and Y. Liu (2016). Recent drag reduction developments derived from different biological functional surfaces: a review. Journal of Mechanics in Medicine and Biology 16(02), 1630001.

Martin, S. and B. Bhushan (2014). Fluid flow analysis of a shark-inspired microstructure. Journal of Fluid Mechanics 756, 5-29.

Marusic, I., K. Chauhan, V. Kulandaivelu, and N. Hutchins (2015). Evolution of zero-pressuregradient boundary layers from different tripping conditions. Journal of Fluid Mechanics 783, 379-411.

Monfared, M., M. Alidoostan, and B. Saranjam (2019). Experimental study on the friction drag reduction of superhydrophobic surfaces in closed channel flow. Journal of Applied Fluid Mechanics 12(1).

Ng, J., R. Jaiman, and T. Lim (2016). Direct numerical simulations of riblets in a fullydeveloped turbulent channel flow: Effects of geometry. In Advances in Computation, Modeling and Control of Transitional and Turbulent Flows, 155-165. World Scientific.

Peet, Y. and P. Sagaut (2009). Theoretical prediction of turbulent skin friction on geometrically complex surfaces. Physics of Fluids 21(10), 105105.
Peet, Y., P. Sagaut, and Y. Charron (2009). Pressure loss reduction in hydrogen pipelines by surface restructuring. international journal of hydrogen energy 34(21), 8964-8973.

Peet, Y., P. Sagaut, and Y. Charron (2010). Turbulent drag reduction using threedimensional structured surfaces. Journal of Fluid Engineering.

Tang, Z., N. Jiang, X. Zheng, and Y. Wu (2016). Bursting process of large-and small-scale structures in turbulent boundary layer perturbed by a cylinder roughness element. Experiments in Fluids 57(5), 79.

Vinuesa, R., M. Hites, C. Wark, and H. Nagib (2015). Documentation of the role of large-scale structures in the bursting process in turbulent boundary layers. Physics of Fluids 27(10), 105107.

Viswanath, P. (2002). Aircraft viscous drag reduction using riblets. Progress in Aerospace Sciences 38(6-7), 571-600.

Walsh, M. (1982). Turbulent boundary layer drag reduction using riblets. In 20th aerospace sciences meeting, 169.

Walsh, M. and A. Lindemann (1984). Optimization and application of riblets for turbulent drag reduction. In 22nd Aerospace Sciences Meeting, 347.

Wen, L., J. C. Weaver, and G. V. Lauder (2014). Biomimetic shark skin: design, fabrication and hydrodynamic function. Journal of Experimental Biology 217(10), 1656-1666.

Wu, J. Z., H. Y. Ma, and M.-D. Zhou (2007). Vorticity and vortex dynamics. Springer Science \& Business Media.

Wu, J. Z., H. Y. Ma, and M.-D. Zhou (2015). Vortical flows, Volume 28. Springer.

Yu, Y., X. Ren, X. Li, and C. Gu (2018). Flow simulation of various riblet shapes and a uniform model for boundary layer. Journal of Applied Fluid Mechanics 11(3). 